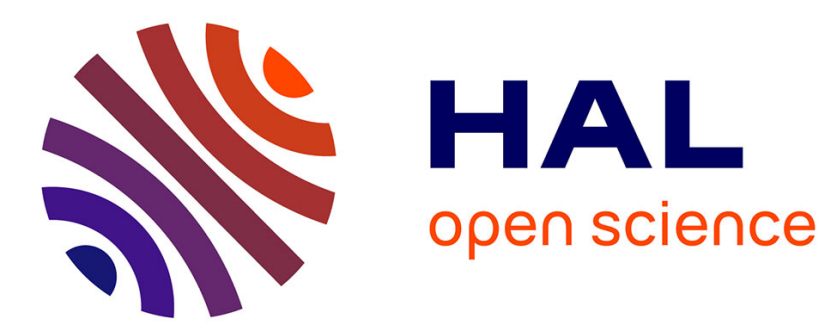

\title{
Vivre le patrimoine urbain au quotidien: pour une approche de la patrimonialité
}

Anne Watremez

\section{To cite this version:}

Anne Watremez. Vivre le patrimoine urbain au quotidien: pour une approche de la patrimonialité.

Culture et Musées, 2008, 11, p 11-35. hal-00603185

\section{HAL Id: hal-00603185 \\ https://hal.science/hal-00603185}

Submitted on 24 Jun 2011

HAL is a multi-disciplinary open access archive for the deposit and dissemination of scientific research documents, whether they are published or not. The documents may come from teaching and research institutions in France or abroad, or from public or private research centers.
L'archive ouverte pluridisciplinaire HAL, est destinée au dépôt et à la diffusion de documents scientifiques de niveau recherche, publiés ou non, émanant des établissements d'enseignement et de recherche français ou étrangers, des laboratoires publics ou privés. 
CULTURE \& MUSEES

Revue internationale

Muséologie et recherches sur hatture

R É D A C T E U R S

Directeur de la publication

Universite Paris-X-Nanterre

JFAN DANALION

COMITÉ DE RÉ DACTION

ANDRÉ DHSSNALIEEES, CON BERNARD SCHEELL, université du Québec à Montréal DANIE. JACOBl, universitê d'Avignon

DOMINIQUE POUl.oT, université Paris-1-Panthéon-Sorbonne

ETH CAll.1.T, Muséum national d'bistoire naturelle

EManuel Enis, univershe d Avignon

JEAN.LOUIS FABIANI, EHHESS

JEAN-PIERRE ESQUÉNAZI, univeritế Jean-Moulin-Lyon-III

JOËLLE LE MAREC, ENSSLSH LyOn

MICHEL RAUTENBERG, université des sciences et tecbnologies de Lille

VES JFANNERET, CELSA Paris-VI-Sorbonne nouvelle

YVES WINKIN, ENS-LSH Lyon

CORRESPONDANTS ÉTRANGERS

Belgique : PHLLPPE VERHAGGEN, université de Louvain-la-Neuve

Italie : EMMA NARD, université de Rome 3

Portugal : JOSÉ AZEVEDO, université de Porto

Espagne : XAVIFR RoIGরé, université de Barcelone
: RAYMOND MONTPHTTT, université du Québec à Montréal

Angleterre : DAVD UZzEL, université du Surrey

Allemagne : HOLGFR HOFGE, université d'Oldenbour
C ULTURE et MUSÉ E S

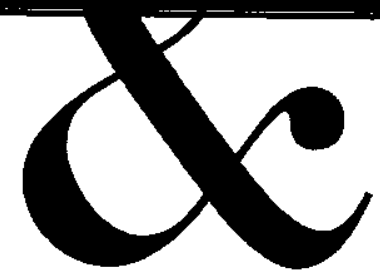

Les manuscrits et toute correspondance doivent être adressés au secrétariat de la rédaction :
Secrétariat Culture \& Musées, Laboratoire Culnute

$\&$ Communication Courrier électronique : pascale.bachevalier@univ-avignon.fr

Conception graphique : PIERRE DUSSER ÉCRAN / PAPIER

Éditeur: Actes Sud, BP 38, 13633 Arles CFDEX (www actes-sud. fr) Achevé d'imprimer en juin 2008

Impression : Imprimerie Normandie Roto

ISBN : 978-2-7427-7646-7 - Dépôt légal : juillet 2008 


\section{S OMMAIRE}

11

Vivre le patrimoine urbain au quotidien pour une approche de la patrimonialité ANNE WATREMEZ

37

La librairie de musée en tant que médium Mathilde Gautier

\section{9}

Un agir communicationnel propre à l'action culturelle la médiation culturelle confrontée au phénomène de l'illettrisme MARIE-CHRISTINE BORDEAUX

R I N C E S

E T P O I N T S D E VUE

Les titres d'expositions : sur quoi communiquent les musées Marie-Sylvie POli \& HaNa GotTESDIENER

Avignon : la cité cinéphilique EMMANUEL ETHIS

Le retour des copistes au musée : un événement? GÉRARD MONNIER E T U P S 101

\section{A $\mathbf{H} \mathbf{I} \mathbf{E}$ R}

Préserver le patrimoine urbain : pourquoi, pour qui, comment ? Sous la direction de Heloîsa Helena Costa 


\section{VIVRE LE PATRIMOINE URBAIN}

AU QUOTIDIEN :

POUR UNE APPROCHE

DE LA PATRIMONIALITÉ

\section{$\mathrm{D}$} $\mathrm{n}^{\circ} 1$ consacré aux nouveaux regards sur le patrimoine (2003), Jean Davallon développait l'idée que la conception du patrimoine la plus communément admise - celle communement admise - celle qui l'ont produite vers nous qui en sommes héritiers ne rendait pas facilement compte des processus sociaux à l'œuvre dans le patrimoine culturel ou natupatrimoine culturel ou natu-
rel, dans un patrimoine collectif et non privé (2003).

Le patrimoine est désormais compris non comme un ensemble d'objets matériels mais comme un phénomène social. Ia relation sémiotique de l'objet patrimonial à ce qu'il signifie est de nature indicielle - il est la résultante physique du monde monument et nous, entre le passé et le présent -, il résulte donc d'une construction symbolique. Le processus de patrimonialisation est aujourd'hui un phenomène largement expliqué (Davallon, he ont été développées : la dimension politique de cette patrimonialisation et la manière dont le patrimoine devient un enjeu touristique (Rautenberg, 1998), sa dimension médiatique à travers l'analyse de l'histoire de récits requalifiant l'objet patrimoine (Tardy, 2003), l'étude d'objets sensibles dans la patrimocommunautés). Ces recherches dans le champ des sciences humaines et sociales - et plus particulièrement celui des sciences de l'information et de la communication - s'attachent à comprendre comment la société construit son patrimoine. Cependant, peu de recherches se posent cette question apparemment vit-on un patrimoine au quotidien dans un espace urbain spécifique, le centre historique ? Comment l'habitant 
vit une ville dite patrimoniale à travers la pratique de l'ordinaire, en dehors de toute pratique touristique? Finalement, en quoi une pratique ordinaire de la ville peut-elle modifier inventer du patrimoine? En quoi changerait-elle son statut symbolique?

Ainsi nous posons ici les bases de la difficile opérationnalisation d'un concept, celui de patrimonialité2 - que nous désignons comme le rapport d'attachement des pratiquants ordinaires d'une ville avec ce qu'ils considèrent comme leur patrimoine par la mise en place d'une méthodologie qualitative. Celle-ci se veut une construction théorique pour comprendre la formation ordinaire du regard patrimonial dans un espace urbain particulier, le centre historique de la ville d'Avignon 3 .

\section{COMMENT PEUT-ON \\ CONSTRUIRE UNE \\ APPROCHE DE LA \\ PATRIMONIALITTÉ}

$\mathrm{P}$

question du lien entre construction du regard patrimonial et pratique ordinaire de la ville perme de renouveler les approches communicationnelles du patrimoine et réaffirme l'importance de l'individu et de son expérience dans cette construction symboliquét. Des chercheurs travaillent depuis quelques années sur la dimension expérientielle et événementielle des processus patrimoniaux pour en révéler leurs mutation t'expérience, devenue centrale, valorisen révéler leurs mutations. L'expérience, devenue centrale, valoriserait le vécu du visiteur en insistant sur le caractère exceptionnel et unique de son passage. Comme le dit Raymond Montpetit, le visiteur doit éprouver, s'essayer, s'exposer aux choses, il s'agit d'en consacrer son caractère exceptionnel. Il devient " comme un consommateur d'expériences, comme un aventurier volontaire acceptant l'invitation entrer dans une quête proposée par ce que l'on nomme la nouvelle économie "(Montpetit, 2005: 111). Toujours selon cet auteur, l'expérience des sites patrimoniaux conduit à trois types de perception : premièrement le plaisir de l'ambiance d'etre qui amène à la contemplation de la valeur d'anciennets; ; deuzmement le plaisir de déambuler : le visiteur a travers un mement le plaisir de déambuler : le visiteur à travers un parcours perçoit la succession des artefacts anciens ; troisièmement le plaisir de regarder des choses. Que penser alors de ces niveaux de perception non plus dans le cadre de la visite patrimoniale mais dans celui de la déambulation urbaine de la pratique ordinaire de la ville? Dans notre cas, l'expériencé 6 ne s'achète pas comme un service, résultat d'une mise en marche des sites patriconme un service, résultat dune mise en marché des sites patrimoniaux, elle n'appartient pas au champ de la production de valeur économique touristique.

D'autres chercheurs ont, par ailleurs, travaillé récemment sur les émotions patrimoniales mais liées à des événements 7 (une catastrophe, une disparition, une redécouverte). Or, on ne peut réduire l'émotion patrimoniale à sa dimension événementielle qui est visible et entraîne des régimes de l'action. Que se passe-t-il quand elle est saisie à travers le régime de l'ordinaire ? L'émotion patrimoniale est alors diffuse et ténue, et difficile à appréhender, à " traquer ".

ÉTUDIER LE PATRIMOINE À TRAVERS LA PRATIQUE ORDINAIRE DES HABITANTS

Nous souhaitons ainsi procéder à une certaine " poétique " du patrimoine, à la croisée entre sociologie urbaine et sémiologie, poétique comprise non dans le sens de Pascal Sansot qui accorde une place importante aux reveries des citadins ct à l'expressivité des lieux, mais " au sens étymologique de fabrication $^{8}$, d'entrecroisement de perspectives, d'objets, de pratiques et de signes "produisant une certaine patrimonialité (Jarrigeon, 2005 : 2). La patrimonialité est un ensemble de représentations patrimoniales liées à l'expérience, au point de vue où le sujet ex, construit par les habitants dans une pratique ordinaire. Flle est la manière de caractériser la relation que ceux-ci ont avec la ville patrimoniale, elle est une mise en relation entre les représentations, les pratiques, les mémoires visà-vis de l'espace urbain. La patrimonialité développe un rapport subjectif aux lieux et à l'espace urbain, à la manière de ce que Michel Rautenberg appelle le patrimoine par appropriation, aui acquiert sa qualité patrimoniale non par injonction de la puissance publique ou la compétence scientifique mais par la démarche de ceux qui se le transmettent et le reconnaissent " (Rautenberg, 1998 : 288). La patrimonialité ne doit donc pas être réduite à ce qu'est le patrimoine par désignation de la puissance publique et doit permettre de comprendre la dimension de l'attachement, dimension que l'on peut aussi désigner comme la construction du regard patrimonial. Il ne s'agit pas de procéder à une étude de réception du patrimoine issu de l'Institution patrimoniale.

La compréhension de la patrimonialité passe donc par la mise en discours de la ville patrimoniale par les habitants, véritables acteurs sociosémiotiques, et provoquée par le chercheur en situation.

1) S'intéresser au pratiquant ordinaire et à ses pratiques

Pour interroger le lien entre les habitants et leur patrimoine, nous nous focalisons sur la pratique ordinaire du centre historique urbain (ou ville patrimoniale). La pratique ordinaire relève du normal, du banal, de l'habituel, dans l'activité de la ville. L'ordinaire peut être quotidien (l'activité se répète tous les jours) ou pas. Il s'agit de ces trajets que l'on fait pour se rendre de son 
domicile à son lieu de travail, des trajets effectués dans le cadre d'une balade familiale ou individuelle, ceux effectués quand on était enfant pour se rendre à son terrain de jeux, à l'école (le che min de l'école est alors le chemin des calades, ces pavés dont jeu consistait à les avoir à portée de pied), etc. Il sagi pratique ordinaire de la ville présente et passee. Avignon pratique ordinaire de la ville présente et passeee. Avignon étant une ville patrimoniale héritière d'un tissu médiéval étroit, la plupart de ces trajets se font à pied.

La notion de pratiquant ordinaire a été reprise chez JeanFrançois Augoyard et Michel de Certeau, puis enrichie. Nous désignons comme pratiquants ordinaires les habitants et les amateurs" qui ont une pratique ordinaire du centre historique, en dehors de toute pratique touristique. Il s'agit d'amener le pratiquant ordinaire à verbaliser ses pratiques ordinaires de la ville patrimoniale et à verbaliser ses pratiques ordinaires de la ville patrimoniale et
de lui faire prendre conscience de pratiques non pensées. Le chercheur et le pratiquant ordinaire co-construisent une mise en récit de l'espace urbain ancien. Les représentations attachées à la ville patrimoniale sont ainsi rendues explicites et deviennent lisibles et visibles.

Ce sont Augoyard et Certeau qui, les premiers, s'intéressent aux pratiques en milieu urbain en décrivant une rhétorique habitante à travers les parcours ordinaires des gens. Ils interrogent " les pratiques de déambulation et de fréquentation des espaces publics. L'objet ce n'est pas les discours sur la ville, ni les descriptions de parcours mais les pratiques d'arpentage de la ville au cours desquelles s'établissent des interactions sociales et des discours " (Mondada, 2000 : 54). Un changement s'est produit dans la façon dont on rend compte de la ville et de sa représentation elle dépend du type de regard sur lequel repose son appréhension et son analyse, et ce regard est passé du point de vue zénithal au point de vue du promeneur. De Certeau, dans L'lnuention du quotidien (1990), nous offre une celebre desions Lionion du quotidien (1990), nous offre une célèbre description, à parti du World Trade Center de New York, des marcheurs dans les rues. Elle montre le passage d'une construction du concept "ville " par les urbanistes appréhendé de façon universelle (le point de vue zénithal) à une construction par les pratiques des populations urbaines, caractérisée par la singularité, la subjectivité, la ruse, la résistance et le détournement (ibid $: 50)$.

En proposant une théorie des pratiques quotidiennes, de l'espace vécu et de la familiarité à la ville à l'aide des outils d'analyse de la sémiotique narrative, Certeau qualifie les pratiquants ordinaires de la ville de " marcheurs dont le corps obéit aux plein et aux déliés d'un texte urbain qu'ils écrivent sans pouvor pleins (Certeau, 1990 : 141). Le marcheur, par ses sans pouvoir lire (Certeau, 1990 : 141). Le marcheur, par ses rhétoriques cheminatoires, transforme en autre chose chaque signifiant spatial. Il sélectionne, "il crée du discontinu soit en opérant des tris dans les signifiants de la langue spatiale, soit en les décalant par l'usage qu'il en fait " (ibid. : 149). Il y a analogie entre l'acte de marcher et l'acte de parler. L'acte de marcher est considéré comme espace d'énonciation : il est un procès d'appropriation du système topographique par le piéton, il est une réalisation spatiale du lieu. L'hypothèse sous jacente à notre questionnement est que la patrimonialité existerait à travers des pratiques ordinaires de l'espace et participerait au sentiment d'appartenance et d'appropriation de la ville. Or les pratiques de l'espace renvoient à des opérations, des manières de faire et à une autre spatialité qui développe une expérience anthropologique, poétique et mythique de l'espace (ibid.).

La notion de pratiquant ordinaire exclut des usagers spécifiques de la ville : les touristes, les commerciaux (qui ont une pratique trop ponctuelle de l'espace de la ville), et les enfants sont des catégories que nous ne prenons pas en compte dans l'échantillon Ainsi, la catégorie " pratiquant ordinaire de la ville "est constituee d'Avignonnais habitant et/ou travaillant dans la ville (l'espace intra-muros et extra-muros) ; des " extérieurs ", c'està-dire des personnes habitant à l'extérieur de la ville (dans le bassin de vie d'Avignon) mais travaillant dans le centre-ville : ils développent une pratique ordinaire, récurrente, voire quotidienne ; des familiers ou " amateurs " de la ville.

2) Les particularités de la notion de pratiquant ordinaire Comment caractériser cette notion liée à l'expérience urbaine ? D'une part en associant le pratiquant ordinaire au fait d'habiter, de résider, de vivre dans la ville. Parler des lieux de sa ville permet de faire dialoguer les aspects publics de la vie individuelle avec des dimensions privées. Mais habiter ne veut pas dire uniquement résider, selon Mathis Stock ${ }^{10}$. Habiter désigne pour lui le fait d'être à la fois physiquement dans un lieu (l'ensemble des pratiques qu'un individu associe à des lieux définit ainsi un mode d'habiter) mais aussi de "rapporter " des pratiques de lieux extérieurs, dans son lieu de vie : "Les êtres humains n'habitent pas seulement un lieu de domicile, n'importe quelle pratique des lieux contribue à l'habiter. Qu'il s'agisse des pratiques touristiques qui associent des lieux du hors-quotidien à des pratiques de récréation, ou des pratiques de loisir, ou du travail ou faire les courses, toutes ces pratiques impliquent pour les personnes d'habiter les lieux. "(Stock, 2004 : 4.) D'autre part, il faut préciser ce que nous entendons par " pratique des lieux " : elle est ce que les individus font avec les lieux et ces manières de pratiquer les lieux ont du sens. Certeau approfondit la notion en faisant de la pratique du lieu le fait de déployer les pratiques pour que le lieu devienne espace : "Un lieu est donc une configuration instantanée de positions. Il implique une indication de stabilité. Il y a espace dès qu'on prend en considération des vecteurs de direction, des quantités de vitesse, des variables de temps. [...] L'espace est un lieu pratiqué. "(Certeau, 1990 : 173.) Le lieu, par la pratique devient espace. 
Une difficulté pour caractériser cette figure tient aux spécificités de la patrimonialité : elle a une part d'impensé. L'ordinaire, le quotidien, le récurrent sont apparemment invisibles. Il est bien connu que nous ne faisons pas attention à ce qui nous entore tous les jours, on ne voit pas ce qui nous est proche, ce quire sous nos yeux. Cet invisible ordini urbain a urbain a éte mis en lumière par Paul Veyne (1988) quand il décrit les attitudes des habitants de la Rome antique avec la colonne Trajane : presque personne ne fait attention à Rome aux détails de la frise gravés à plus de trente mètres de hauteur, pourtant la colonne est un lieu de rendez-vous, de promenade. Cette attitude ne signifie pas qu'elle n'a pas d'importance pour les habitants mais que la colonne s'est fondue dans le paysage les habitants les pratiques quotidiennes. Comment lo paysage, les usages et es pratiques quotidiennes. Comment donc rendre visible, lisible et sensible cet invisible ordinaire ? L'entretien à domicile ne pouvant rendre compte seul de cette patrimonialité ténue et quasi impensée, l'itinérance in situ a été choisie car elle a l'avantage de ne pas " tromper " en ce sens où l'enquêté, devant l'acte de faire, est obligé de mettre en discours sa pratique et de la rendre verbalisable. A côté de cette invisibilité ordinaire, le pratiquant ordinaire connaît, par une pratique de l'ailleurs, à l'étranger ou dans d'autres villes, une expérience patrimoniale mais sous le mode de la visite. Son regard patrimonial se construit donc ausi aille de lors de visites touristiques et il le ramèn donc aussi ailleurs construction qui se fait ailleurs a-t-elle une influence sur sa pratique de la ville ? Y a-til reurs a praique de la ville ? Y a-t-il réutilisation de ce qu'on a vu ailleurs pour le réinvestir ici ? L'expérience vécue ailleurs produit-elle, à long terme, un effet sur les expériences futures dans un flux continu d'expériences ?

Pour saisir la totalité du concept de patrimonialité, il est nécessaire de se focaliser sur les individus afin de dégager des indicateurs de patrimonialité issus de l'expérience individuelle, pour ensuite aller au-delà et dégager des régularités à dimension sociale. La patrimonialité est donc un construit pour comprendre le regard patrimonial ordinaire. Elle doit être comprise comme une mediation - en tant qu'entre-deux-mondes - entre l'univers individuel et subjectif de l'expérience et l'univers collectif symbolique de la patrimonialisation, et il $y$ aunit plus ouctif et des glissements vatrimonialisation, et il y aurait plus ou moins des glissements vers l'un ou l'autre des pôles.

3) La nécessité de considérer l'expérience sensible de la ville. Considérer que la pratique ordinaire de la ville a une influence sur la construction du regard patrimonial suppose ainsi trois postulats : le premier est que l'expérience sensible de la ville est située, le second est qu'il y a engagement du sujet percevant le milieu social et les qualités physiques et sensibles de l'environnement, le troisième est qu'il y a mise en jeu des sens, du corps, de la motricité du citadin. La pratique ordinaire de la ville permet l'expérience sensible de la ville. Qu'entendons-nous par expérience sensible de la ville ? Jean-François Augoyard la définit à travers deux phénomènes : percevoir et exprimer une ambiance. Ainsi la ville est donnée à voir, à déchiffrer, à consommer parce que dense de signes. Flle est un système de signification qui s'elabore à travers sa bore à travers sa spatialité, les pratiques d'espace mais aussi le discours publics et privés qui la traversent et qui la configurent. Le rapport que les citadins établissent avec la ville est un rapport de décodage (Lynch, 1971) dont le chercheur doit provoquer la compréhension.

La ville patrimoniale constitue ainsi un texte en ce sens qu'elle est une mise en discours par les pratiquants ordinaires et provoquée par le chercheur. Selon un usage courant en sémiotique, la notion de texte désigne " l'ensemble des éléments signifiants d'un objet, sans se réduire au cas particulier du texte linguistique " (Dondero, 2007). Mais l'espace urbain n'est pas seulement un pur champ de déploiement de l'activité du sujet, il existe des règles silencieuses, des articulations sociales qui territorialisent l'espace social. Les formes urbaines ont ainsi la capacité de manifester du social. Les formes urbaines ont ainsi la capacité de manifester du
sens. Cette puissance sociale de l'espace, Maurice Halbwachs en sens. Cette puissance sociale de l'espace, Maurice Halbwachs en
fait le fondement de la mémoire et de l'expérience urbaine ${ }^{11}$. L'espace urbain, par sa nature appelle un rapport proxémique avec ses pratiquants et ce qui le compose (espaces, rues, monuments, etc.). Et le pratiquant ordinaire est amené à composer avec l'environnement, son parcours est contraint par la présence d'autres membres. L'espace urbain est une scène permanente de
sociabilité.

UNE METHODOLOGIE QUALITATIVE QUI FAIT VERBALISER LE PRATIQUANT ORDINAIRE

Au cœur de la recherche il y a la nécessité d'un questionnement méthodologique. La méthode est de type qualitative * clinique ", c'est-à-dire qu'elle observe des sujets et des situation individuelles. Nous avons exploré un certain nombre d'outils permettant de repérer et d'étudier la patrimonialité et ses expressions, ses modalités d'existence dans les discours et les pratiques ordinaires de l'espace urbain.

1) L'opportunité d'un terrain " fort ": Avignon, une ville patrimoniale.

Le terrain de la recherche est la ville d'Avignon, plus particulièrement son centre historique. Celle-ci a une personnalité spatiale forte : construite sur un rocher (le rocher des Doms) au pied duquel se trouve un fleuve (le Rhône), elle est une ville patrimoniale : ville remparts, le centre-ville historique est situé dans un intra-muros - héritage du tissu urbain médiéval européen - 
opposé à un extra-muros qui regroupe les autres quartiers principalement construits dans la deuxième moitié du xxe siècle. L'intra-muros est un périmètre urbain protégé par la loi (un secteur sauvegardé), une partie de son patrimoine est inscrite au patrimoine mondial depuis 1995 (l'ensemble monumental pontifical), il est ce que nous appelons ici le centre historique. possède un patrimoine monumental exceptionnel, caractétse par une richesse architecturale de monuments construits du xIV a XVIIJe siècle heri xents, élises, vents, eglises, livrées, hôpitaux, hôtels particuliers). Ce caractère patrimonial a des conséquences dans les différentes pratiques de la ville parce que le patrimoine fait partie intégrante de l'espace, de son identité urbaine. Les institutions municipales ont développé une politique culturelle autour d'une "image de marque pat moniale " axée sur deux "monuments ": le palais des Papes / le pont d'Avignon inscrit au patrimoine mondial et le festival international de théâtre créé par Jean Vilar. Par ailleurs, le rôle culturel et identitaire du centre historique d'Avignon dépasse le poids démographique et économique; son importance sociale, culturelle et symbolique est primordiale. L'intra-muros a résume ct a identifie "la ville, il est la mise en représentation culturelle de l'espace, il est un résumé de l'identité de la ville.

2) Une recherche qui repère le caractère ténu et impensé de la patrimonialité

Nous avons développé une approche scientifique à une échelle microsociologique, en sortant des approches normatives, holistiques et décontextualisées, pour aller vers le particulier, à la manière de la micro-histoire ${ }^{12}$. Notre posture de recherche prête une attention parmicro-histoire ${ }^{12}$. Notre posture de recherche prête une attention particulière aux témoignages des différents acteurs pour les relier aux contextes dont ils participent. Ce type d'approche doit permettre de déceler des processus fins, flous et difficiles à appréhender, caractéristiques principales de la patrimonialité en ce sens qu'elle constitue une représentation construite dans un rapport sensible au monde qui l'environne. L'étude de la patrimonialité nécessite ainsi une méthodologie fine car elle est constituée de ce que Sansot appelle des "signes légers" caractéristiques de la dimension sensible de cet objet, ce qui la différencie selon nous de la patrimonialisation, construite sur du savoir scientifique et historique. Il y a ainsi nécessité de considérer la sollicitation des sens, la percepton du sensible dans l'étude de la patrimonialié car, sen Jean-jacques ensible dans l'étude de la patrimonialité car, selon Jean-Jacques Boutaud ${ }^{13}$, la dimension sensible des signes est sous-estimée dan les processus de significations.

La recherche utilise les acquis de l'ethnographie urbaine qui naît de l'exigence d'observer les pratiques sociales et culturrlles urbaines dans la diversité de leurs contextes (Mondada, $2003: 24$ ). À l'instar d'Ulf Hannerz nous envisageons une certane an : 24). pologie de la ville qui se concentre "sur des phénomènes et des lieux constitutifs du caractère urbain de la ville " notamment les espaces publics, les monuments, les images de la ville (Hannerz, 1983). Cette posture implique que le chercheur soit présent dans la ville.

Nous avons exploré un certain nombre d'outils permettant de repérer et d'étudier la patrimonialité et ses expressions, ses modalités d'existence dans les discours et les pratiques ordinaires de l'espace urbain. Nous avons choisi de réaliser des entretiens itinérants sur l'ensemble du centre historique de la ville d'Avignon. Ils sont considérés comme un dispositif - en tant que tout agencement d'éléments humains ou matériels réalisé en fonction d'un but à atteindre (Jacquinot-Delaunay \& Monnoyer, $2000: 10$ ) - à valeur heuristique, permettant de comprendre in situ, et en direct, comment le regard patrimonial ordinaire se construit.

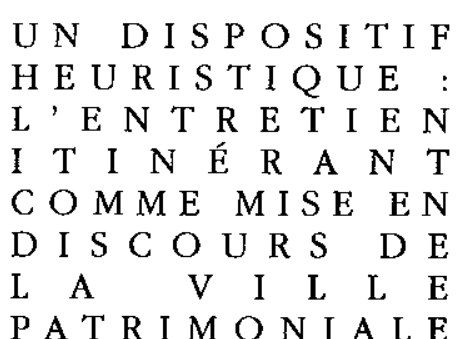

UNE TRADITION DE L'ANALYSE DES PARCOURS DANS L'ESPACE

Ce dispositif de l'itinérance qui permet au chercheur d'être en situation n'est pas inédit et a été utilisé dans d'autres disciplines (psychologie, muséologie, sociologie urbaine) sur des objets différents, comme celui d'espace public. Dès les années 1920, des chercheurs tentent d'évaluer les lieux publics en étudiant les parcours. Ces études seront reprises régulièrement à partir des années 1980. En muséologie, ce sont Eliséo Véron et Martine Levasseur qui popularisent ce type d'évaluation grâce à leur bestiaire ${ }^{14}$ : ils construisent ainsi des types de visite en fonction de critères sociologiques et en arrivent à développer une ethnographie de l'exposition. Sophie Mariani-Rousset, dans une thèse en psychologie (1992), s'intéresse aux parcours dans l'exposition en tant que révélateurs de comportements et de constructions de sens. Ce qui intéresse cet auteur dans l'objet exposition est sa dimension d'espace public et l'appropriation qu'un visiteur se fait de cet espace pendant le moment de visite. Ainsi * l'appropriation du contenu de l'exposition se concrétise dans celle de l'espace qui se manifeste par les parcours de visiteurs " (Mariani-Rousset, 2001 : 29). Dans les deux recherches présentées, l'évaluation se fait principalement par le suivi de parcours et la technique de l'observation in situ, basée sur une grille d'observation détaillée, 
le visiteur ne sachant pas qu'il est suivi. Un entretien peut être sollicité mais il est séparé de l'observation et se déroule avant ou après celle-ci. Ce sont ainsi les comportements qui sont observés, l'acte de parole n'est pas sollicité. De plus, l'exposition, en tant que média n'est pas un objet d'analyse anodin et implique, par la nature de ce dispositif, des constructions de sens spécifiques : le parcours de visite observé constitue en fait trois parcours : le parcours pensé par le concepteur, le parcours proposé par la mise en exposition et le parcours vécu par le visiteur.

C'est surtout la sociologie urbaine qui a développé ce type d'enquête par entretien itinérant ou parcours commenté, particulièrement riche quand on étudie l'espace de la ville. L'itinérance est considéree par Mondada comme le mode privilégié de linéarisation et de mise en perspective de la ville. Ce mode d'écriture de la ville va souvent de pair avec un choix méthodologique qui privilégie une approche "micro " (Mondada, $2000: 49$ ). Augoyard, depuis ses débuts, s'est intéressé aux rhétoriques cheminatoires comme l'expression habitante constitutive de l'expérience sensible de la ville et comme témoins des manières de la percevoir. Son objectif est de saisir l'espace vécu et la pratique des habition que ceux-ci donnent de leurs parcours et de leurs cheminements (Augoyard, 1979). La vie quotidienne, à travers la pratique des cheminements, aurait l'aspect d'un langage. Les pas énonceraient des actions spatio-temporelles dont la configuration d'ensemble aurait un style. L'organisation de ces styles propres à chaque habitant constitue une rhétorique cheminatoire. Il faut alors relever les figures de cette rhétorique et les genres de combinaison qu'elles composent 15

Au fil de ses recherches, Augoyard a élaboré une technique d'enquête sur les manières d'habiter, la conduite de récit. Ce dispositif allie la narration et la spatialité de la ville pour comprendre comment l'habitant crée son sens de la ville en marchant. Le paradigme de la conduite de récit (Augoyard, 2001) est construit sur l'appel à la mémoire quand on interroge l'habitant sur ses pratiques quotidiennes. Le perceptible est ainsi mémorable, qui est lui-même narrable. À côté de ce moment d'entretien narré - récits individuels -, le chercheur fait appel aux cheminements. C'est cette articulation entre le parler et le cheminer qui nous a particulièrement intéressée. Parce que l'habitant n'existe pas abstraitement, le cheminer est une des manières de comprendre le mode d'être dans l'espace, mode à la fois très quotidien et très dynamique. "Marcher, c'est mettre la ville en emploi du temps vécu. "(Augoyard, $2001:$ 178.) La quotidienneté, par ses va-et-vient, donne à la ville une certaine allure, sa manière d'apparaître.
La terminologie d'entretiens itinérants sera préférée à celle de parcours ${ }^{16}$ : dans notre cas, le dire a autant d'importance que le faire. La notion de parcours (chemin, circuit, itinéraire, trajet, cheminement, course, traversée, etc.), utilisée en muséologie, implique d'être à la croisée entre l'utilisateur et le concepteur 'est l'utilisation par l'un organisé par l'autre (Mariani-Rousset, cest). Litinerant 1992). L'itinérance implique quant à elle un processus de décision dans le rôle endossé par les participants et uniquement par eux. Le chercheur s'efface devant l'enquêté.

Notre dispositif est fondamentalement différent de cette tradition, son atout principal étant de dire et faire en même temps et d'être une construction de l'interviewé. Il prend en compte l'espace ou le territoire de la ville du pratiquant. La situation de l'enpace ou le territoire de la ville du pratiquant. La situation de l'entretien se superpose à l'activité effectuée in situ et influence
l'économie de l'échange entre enquêteur et enquêté. Le rôle de l'économie de l'échange entre enquêteur et enquêté. Le rôle de
l'enquêteur s'efface le plus possible pour influencer le moins possible les logiques de parcours et d'interprétation. L'entretien itinérant est un entrelacs du percevoir et du dire. Sa mise en place est possible si on peut appréhender la perception à partir de ce qui est rapporté verbalement (Thibaud, $2001: 82$ ). Le déplacement sur est rapporté verbalement (Thibaud, $2001: 82$ ). Le déplacement sur
le territoire, sur l'univers de référence du pratiquant ordinaire de la le territoire, sur l'univers de référence du pratiquant ordinaire de la
ville accentue la subjectivité de la situation d'enquête. L'enquêteur devient un témoin : il certifie l'authenticité du discours (Vareille, 2001). Il apporte de même un éclairage sur les conditions de production de ce dispositif. L'entretien itinérant prend ainsi en compte la dynamique du lieu et ses différentes pratiques.

L'entretien itinérant tire sa force de la mise en situation du chercheur et de la simultanéité du parler et du marcher in situ. Il ne s'agit donc pas pour l'interviewé de réfléchir à ses trajets quotidiens présents et passés en faisant uniquement appel à ses souvenirs mais de faire ses trajets en direct. Le dispositif des entretiens itinérants permet de faire jouer pleinement à l'espace urbain son rôle et de saisir in situ ses effets. La présence physique du pratiquant ordinaire dans l'espace engendre des événements sensibles à gérer affectivement. Refaire les pas dans la ville, en même temps que l'acte de narration, multiplie la vérité des lieux. L'enquête en situation permet certes “ un discours sur le lieu ", où,

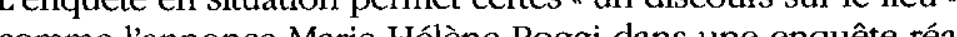
réalisée sur le quartier du Marais (Poggi, 1999), l'espace est le référent dominant proposé par l'enquêteur. Ces discours " sur " permettent alors l'émergence de la mémoire individuelle, d'avoir accès aux expériences fondatrices de l'identité sociale et culturelle des enquêtés (Poggi, 1999 : 368). Mais l'enquête en situation permet aussi d'obtenir un discours " dans " le lieu " où l'espace n'est plus aussi d'obtenir un discours " dans " le lieu " où l'espace n'est plus jeulement le référent mais en même temps la composante ma-
jeure dituation de production du discours dont l'enquêté a 
la maîtrise "( ibid.). Marie-Hélène Poggi parle alors d'effet d'anameffet particulier que provoque le lieu et Lors de l'itinéte force de la preuve.

Lors de l'itinérance choisie par le pratiquant ordinaire, il commente ce qui s'offre à lui. Le rythme des parcours ou de l'itine rance dans l'espace urbain est celui de l'entretien, le de l'itinése fait dans l'instantanéité, il est spontané nous avons accès à des discours sur le patrimoin l'acte de parole parcours issus de l'itinérance sont une patrimoine, et les différents plusieurs énonciations pié sont une mise en superposition de cette méthonciations piétonnières. Selon Jean-Paul Thibaud, cette méthode a un double mérite : elle permet d'associer un ensemble de jugements donnés par un sujet à une pratique un déplacement qui doit intégrer une appropriatione pratique de de ce qui le constitue ; elle permet que l'ension de l'espace ments produits ne soit pas uniquement lensemble des jugeperspective des connaissances, qu'un individu connaissances, savoirs, présupposés, souvenir le discours produit est le discours produit est une construction qui intègre l'enseme des proprietes sensibles d'un environnement, d'un lieusemble action de ces propriétés à la pratique ordinaire. Nous et l'interrons le sensible comme embrayeur de considélocales comme motifs a

Concrètement, peut être exécuté soit dans procède-t-on ? L'entretien itinérant peut etre execute soit dans la suite d'un entretien ponctuel avec une meme personne : on a alors deux discours dans deux situations differentes. On peut percevoir des décalages deux situa(l'entretien ponctuel) et le faire (l'entretien itines entre le dire être réalisé de manière inédite avec un itinérant). Soit il peut contacté préa tif avant de réaliement. Dans tous les cas, expliquer le dispositif avant de réaliser l'entretien itinérant est primordial et permet d'établir un contrat de communication : la consigne ret permet complexe, la durée de l'itinérance (entre une hne relativement deux heures et demie), l'autorisation de l'enregistre et quart et tretien, la reproduction du trisation de l'enregistrement de l'enLe délai entre le moment celui de l'entretien est réfléchir au parcous souvent mis à profit par l'interviewé pour entech qu'il choisit de nous faire faire. Nous avon donc des parcours qui ne sont pas spontanés, l'interviews avon fixé "les grandes lignes" du parcours lors de contre. Ce genre de dispositif réintroduit l"insta première renl'économie du dispositif. Face au sentimit linstance affective dan demandée de la part du chose à l'interviewé a l'interviewé et de l'encourager au début de l'entretien. fausse banalité est justement une richesse puisqu'on a des ch. Cette d'obtenir des récits véridiques. Au fur et à mqu'on a des chances l'enquêté oublie la situation provoquée et se libère de l'entretien, Il s'agit aussi de fuire

rôle de guide et de lui fairesser à l'enquête, pour un temps, le la ville. Selon Antoine Hennion, l'amateur est " un virtuose de l'expérimentation esthétique, sociale, technique, corporelle mentale, il est le modèle même d'un acteur inventif, réflexif, obligé de remettre sans cesse en cause les conditions et moyens favorables aux effets qu'il recherche " (Hennion, 2003: 283). L'hobitantamateur construit ainsi son propre itinéraire et devient expert, il n'est plus réduit à occuper une place fixe et limitée.

Comment amener le pratiquant à parler de sa pratique ordinaire de la ville et de son rapport au patrimoine qui l'entoure? I s'agit de créer les conditions pour obtenir du récit et de la description afin de repérer des traces et des modes d'existence de patrimonialité. Raconter sa ville permet de faire apparâtre la émotions et expériences patrimoniales. Quant à l'action de décrire ce que les interviewés considèrent comm laction de moine, elle permet d'aview moine, elle permet d'avoir accès à des registres de valeur. Comment alors formuler des questionnements pour que le pratiquant ordinaire parle de son patrimoine sans qu'il s'en rende compte? Ainsi nous commençons généralement par demander aux gens de nous emmener sur les lieux qu'ils pratiquent, apprécient particulièrement, quir leur evoquent un souven qu'th une expérience, les lieux quils fon visiogu à une experience, les lieux qu'ils font visiter à leurs amis quand on leur rend visite. On leur demande de refaire leurs trajets quotidiens (du domicile au lieu de travail par exemple). Le mot patrimoine est évité car il est trop connoté " monument " et désigne des objets patrimoniaux reconnus. Il "sonument " et désigne lances, d'approfondir certains propos. Dès la form dation deconsigne, les représentations que les interviewes ont de la moine sont representations que les interviewés ont du patrimoine sont à l'œuvre. En effet, ils font une différence forte entre le patrimoine qualifié d'objectif, le patrimoine national (le palais des Papes) et les lieux forts qualifiés de proustiens (en référence à sa madeleine) mais non patrimoniaux car issus de leur rience privée, individuelle et donc non communicableur expégeable. Ce patrimoine issu de la pratique de la famille, partaprivé n'est pas considéré comme ce quique de la famille, intime, privé n'est pas considéré comme ce qui est patrimoine par l'Institution, comme si celle-ci avait raison et eux tort par rapport à ce que doit être la doxa patrimoniale.

LA NARRATION DANS L'ENTRETIEN, UNE PRATIQUE DISCURSIVE POUR COMPRENDRE LES PRATIOUES OUOTIDIENNES.

L'entretien est un événement interactionnel au cours duquel co-construisent une cours duquel monde (Mondada 2000 : 87) Il configure ains les obje de cours, il est une pratique de représentation qul configure données. L'entretien non directif quil se l'échange L'entretien non directif, qu'il s'oriente vers l'interview, lechange conversationnel ou le récit de vie, crée un contexte particulier accompli par les conduites interactionnelles des participants 
qui produisent une parole vers ce contexte et qui répondent ainsi tien, par conséquent, projetées par les uns et les autres, l'entretien, par conséquent, ne peut être lu comme étant un discours valable en tout temps et en tout lieu. Le discours est un discours dynamique, ses contenus recueillis construisent un processus intelligibilités en s'ajustant au contexte construisent leurs propres présence du chercheur est un contexte particulier de l'entretien, la cours (ibid. : 90). Aurs $(i b i d$.

Au coeur de l'entretien, il y a donc un récit, une narration. La narration est une pratique discursive courante et permet d'introduire deux dimensions : celle des pratiques quotidiennes et celle
d'une cohérence propres habitude donnée au parcours pour mettre en intrigue ses pement du personnagesit personnel permet de raconter de faire veveloppersonnages, de rappeler des souvenirs, de de faire vivre des petites histoires privées dans le collectif, de faire partager les de la ville, de se mettre en scène imagée. C'est donc part en scène et créer une communauté tion au monde17 et le prostion que lhomme donne significarécit lie raison et é le processus dinterprétation produit par le humaine autantion. Le récit donne à vivre une expent par le humaine autant qu'à voir. La narration lie et donne expérience des données disparates et à la séquentialité donne cohérence à à-dire à la disposition d'objets décrits le long de l'itercours, c'esttituant un réseau. La narration permet long de l'itinéraire et conspoint de vue, elle apparaiton permet à l'enquêté de soutenir son du sens (ibid.).

LE REGARD PATRIMONIAL ORDINAIRE : QUELQUES

I N D I C A TEUR S

DE PATRIMONIALITÉ

itinérants ? Plusieurs nivment ensuite analyser ces entretiens compte : le premier se fiveaux d'analyses doivent être pris en interviewés. En effet, des portraits des pratiquants ordinaires ville peuvent être dressés a parts de pratiquants ordinaires de la les lieux fréquentés aujou artir de plusieurs éléments comme person personce, la de déplacement, leurs représentations patrier, leurs modes doivent pas être considérés comme une typotrimoniales. Ils ne partir d'individualités, mais plusieurs une typologie construite à des figures. Chaque figure plusieurs portraits peuvent constituer nouer une relation, une objet patrimo rexpérience, un attachement avec de lyse est celui des paronu ou inconnu. Le second niveauec un lyse est celui des parcours effectués dans le centre hisuu d'anasoi et de l'interaction interviewécher entre historique en

VIVRE LE PATRIMOTSE 24 IVRE LE PATRIMOINE URBAN AU QUOTDIEN.
C ULTURE \& MUSÉS No se situe dans l'analyse développés ici. Enfin le troisième niveau des entretiens qui permet repérer quelques indicateurs de patrimonialité concentrant des valeurs, des croyances, des représentations. Nous proposons donner quelques premiers résultats qui permettent de comprendre concrètement ce que recouvre le concept de patrimonialité.

\section{LA PATRIMONIALITÉ EN TANT QUE CADRE DE VIE SENSIBLE FAMILIER}

La patrimonialité est avant tout un attachement qui se fait par la proximité affective et physique de l'objet patrimonial lors de pratique du lieu. Il est vécu en tant qu'objet de familiarité et participe à la vie familiale du pratiquant. Cette relation de proximité est telle qu'il devient comme une personne rution de proximité recherche des moments privilégiés personne humaine. L'usager recherche des moments privilégiés avec lui en se créant des occasions, le soir, la nuit quand il n'y a personne : "Il m'arrive de faire une petite pointe jusqu'au palais par exemple le soir parce que c'est agréable le soir il y a du monde et puis il change de
couleur et puis comme tout se calme on a l'impression pour soi. "(Entretien tout se calme on a l'impression de l'avoir caur soi. "(Entretien $n^{\circ} 6$, femme, cinquante ans.) Ces moments calmes sont aussi des moments hors affluence touristique. L'expérience patrimoniale ordinaire prend ici toute son ampleur : voir par exemple le palais des Papes de sa fenêtre des toilettes, le voir de dos, ou, comme le dit un pratiquant ordinaire, voir son le voir rière ", fait entrer le monument dans la vie quotidienne, comme un copain qui partage votre vie.

La patrimonialité est donc aussi une question de point de vue en tant que posture physique du pratiquant ordinaire qui regarde un objet patrimonial. Il faut voir le lieu, ment que le commun des mortels - les touristes - le voir autreordinaire est fier quand il peut faire découvrir - , le pratiquant vue inédite de l'objet patrimonial. montrent qu'un objet patrimonial. Des extraits d'entretiens nous dans un qu'un objet patrimonial n'existe que parce qu'il est vu dans un contexte particulier, il devient patrimonial pour le pratiquant à un moment précis et pas à un autre. Ici, la patrimonialité s'organiserait à partir du lieu où on est en fonction du pont de vue et de la posture du pratiquant ordinaire et non du point ment en fonction de sa pratique du lieu : On peut pas uniqueje l'apprécie vraiment, le palais du lieu : "On peut pas dire que c'est pas un monument, le palais des Papes, il m'impressionne, c'est pas un monument qui me touche vraiment [...]. Je le voyais de mes toilettes (rires) c'était magnifique, j'aimais bien le voir parce que [ça voulait dire quel j'arrivais à Avignon bien le voir quand les gens arrivaient chez moi ils dient "ou c'est vrai que palais des Papes a des aspects de l'Utopia aussi j'avais bien aimé passer sympathiques du côté mélange avec le quais bien aimé passer derrière, j'aimais bien le de les vivre au qua ça mporte quelque chose de les vivre au quotidien. "(Entretien $n^{\circ} 4$, femme, vingt-huit ans.) 

Ici se produit un glissement à partir du point de vue qui change,
point de vue à la fois comme posture physique triviale (les toi-
lettes) et posture symbolique (sonre phases de la réflexion révèue (son expérience). Les différentes pratiquante de la position de des paliers qui font passer cette qu'elle n'appréciesas) a ce "On doit apprécier " un monument construit à partir de sas) à celle de son propre regard patrimonial tidiente a parti de son experience (le festival et la pratimonial tidienne du lieu). Elle quitte le discours de a doxatique quoqu'un monument patrimonialisé voire patrim doxa qui affirme être impressionnant, beau et sence patrimoine mondial, doit patrimonial est ici construct un objet atrimonialité dans le

Mais la vue peut être aussi panoramique, elle est alors constuctrice d'un paysage organisateur. Avignon s'est contronstrucd'un rocher, le rocher des Doms, Avignon s'est construite autour se situe à l'ouest le Rhône et à l'est l'étalement de cité, au pied duquel de la ville. Le pratiquant ordinire, luatement du centre historique Doms (qui est aujourd'hui un jare, quand il se rend au rocher des cle), procède à uno dur un jardin public paysagé au Xvire siè veut dire prendre crable " montée ", puisque aller au ro veut dire prendre de l'altitude à parti ", puisque aller au rocher

L'envie de hauteur pour à partir du centre-ville.

urbain en un seul coup d'œeil est un ber, par la vue, le paysage tains pratiquants ordinaires de $360^{\circ}$ ", c'est-à-dire monter a de jouir de la vue panter au jardin public situé sur un rocher Roland Barthes, panoramique sur la ville et son arrière-pays. qu' au marthes, dans le très beau texte " La tour Eiffel ", nays. qu'" au merveilleux allégrement de l'altitude, la vision ", nous dit mique ajoutait un pouvoir incomparate, la vision panorad'oiseau [...] donne le monde à lire et dintellection : le vol voir ; [...] le vol d'oiseau permet de tépan seulement à percevoir les choses dans leu pernet de dépasser la sensation et de

Le panoramast "(Barthes, 1993 : 1386-1387). cherchent à déchiffrer, ils essayent d'y ratiquants ordinaires connus, d'identifier des repères. Il permet de se preje des lieux ailleurs, inconnu qu'on se prend à rêver de se projeter vers un veillée par la vue qu'on a sur le Rhôner. "Je suis toujours émerles Avignonnais c'est quelque lhose parce que le Rhône pour saint Bénézet passe dulque chose d'assez important / le pour papes, i'y suis donc attachee c'est lhistoire d'Avignon te pont papes, Jy suis donc attachée c'est mon berceau // parce et de beaucoup de souvenir d'enfance je repère des // parce que j'a avec mon père avec ma mère / (rires). " (Entretien $n^{\circ} 14$, femmer à mon cour panoramique, un moment enme, quatre-vingts ans.) La vision timents collectifs et d'adhe partage, de vivre ensemble, de senaller au roche c'est revive l', cest finalement revenir au berceau de c'est revivre l'histoire de la ville, c'est faire partager un la ville, d'appartenance à la communauté avignonnartager un sentiment ramique est ainsi un moment d'émotion paise. La vision pano26 VIVRE LE PATRIMOINE URBAIN AU QUOTDIEN
CULLTURE: \& MUSÉS No maison, se remémorer des souvenirs d'enfance, se confirmer dans de la ville.

Comment les pratiquants ordinaires décrivent-ils cette proximité affective, cette relation sensible, intime avec l'objet patrimonial ? Elle est liée évidemment à l'émotion mais elle ret patrimonial ? assez vague. "Ressentir un peu le bâti ", ressentir ce trualement plutôt du coup de coeur de l'émotion à un moment sion esthétique passe ainsi par la vue et pement T. La dimenappropriation : passe ainsi par la vue et permet une certaine appropriation : admirer, contempler, profiter de l'ambiance entourant l'objet patrimonial devient vital : "C'est là qu'on a une vue magnifique, quand on était sur la Barthelasse on venait toutes les heures de l'après-midi pour voir les différents benait toutes les chent des flèches ! [...] C'est vrai qu'on aime bien de toui se détaon revient à Avignon le soir on revient toujou de toute façon si ment sur la ment, sur la place du palais des Papes [...]. Quand on rentre d'Utopia on passe toujours par ici et après on se dit qu'on peut rentrer c'est une rencontre privilégiée c'est tellement grandiose et on est tout petit. " (Entretien itinérant $n^{\circ} 1$, couple) Mais la dimension sensible, esthétique passe aussi par des sons, Mais la dimenpratiquant ordinaire de la ville, patrimoniale, est attentif patrimoniale, est attentif aux couleurs, aux jeux d'ombre et de lumière traversant les feuilles des arbres et jouant sur les calades, aux odeurs qui rappellent des souvenirs liés à l'enfance. Ainsi quand cette vieille dame de quatre-vingts ans évoque un lieu Aatrimonial - le rocher des Doms -- des odeurs et des goûts de gâteaux rimonial tent : "Il y avait quand même des marchands d'hosties [..] n remonpas des hosties c'est des oublis, c'etait dans unosties [...] non c'est pas des hosties c'est des oublis, c'était dans un grand machin rond c'était des espèces de galettes très fines qu'on vendait et j'en ai plus jamais vu et tous les enfants, parce que c'était très bon marché, alors on pouvait vous donner des sous pour acheter des oublis voila enfants aiment bien grignoter, ce marchand je m'en rappelle bien me rappelle bien du goût c'était comme des petits-beurres mais beaucoup plus plats. "(Entretien $\mathrm{n}^{\circ} 14$, femme, quatre-vingts ans.)

\section{ÊTRE EN LIEN AVEC LES HUMAINS DU PASSÉ}

Vivre la ville patrimoniale d'Avignon au quotidien c'est aussi se projeter dans sa période faste, celle de la construction du palais
des Papes. L'univers de référence est alors celui du Moyen Âge : on s'interroge sur l'époque, les métiers mobilisés pour les Ana : tiers, les modes de vie, bref, on fait référence à les chan" médiévalité ". "J'aime bien arriver par l'arrière à une certaine culièrement après lorsqu'on suit la rue Peyrolis partil'impression d'être encore au Moyen Âge la rue Peyrolerce qu'on a sous l'arc-boutant oui parce que Á Á la rue Peyrolerie qui passe nous larc-boutant oui parce que c'est à peu près le seul endroit qui nous donne l'illusion d'être à l'époque de la construction du palais. "(Entretien $n^{\circ} 6$, femme, cinquante ans.) 
Les pratiquants ordinaires sont curieux quant à la façon de vivre des prédécesseurs : on joue le jeu en essayant de se mettre dans leur peau, on essaye de se rapprocher du temps originel de ces ancêtres. Un certain nombre d'interviewés évoque le chantier du monument emblématique de cette période le pala le chantier en s'interrogeant sur le travail des ouvriers, nombre de pierres que la construction compte, les artisans, le travail, l'extraction dans les carrières, le tranpte, les méthodes de Le monde des artisans fes carrières, le transport sur le Rhône, etc. fe monde des artisans fascine et invite au respect : "Ce qui me fait plaisir, ce qui me touche ce sont les gens qui ont fait ce truc-là à l'époque, comment ils travaillaient, quels étaient les ce truc-la sociaux entre les gens, en fait c'est un peu un rêve vivant tretien $n^{\circ} 12$, homme, cinquante et un ans.) reve vivant. " (Enhumains qui ont pensé, construit et un ans.) Ce sont des êtres donc des humains du Le respect le seul indice.

Le respect est tel que si l'édifice est abîmé, abandonné ou détruit, on estime que cette attitude est criminelle en ce sens qu'elle ne respecte pas le travail des anciens. On va jens regretter ce savoir-faire qui peut disparaitre: "Rue $\mathrm{Pa}$ sur la gauche il y a un immeuble qui est ancien mais jenl-Sain et pas dire quelle époque mais il y a des // appuis de je ne saurais forgé qui sont Louis XIV et y a des // appuis de fenêtres en fer statue qui sont Louis XIV et qui sont fantastiques, ça va être une statue qui est nichee dans un mur [...] c'est désespérant de voir que toutes les poignées de portes, les sonnettes anciennes voir heurtoirs disparaissent parce qu'ils sont volés, moi je vois, les près puisque les gens viennent me demander de jerp ça de Entretien $n^{\circ} 12$, homme La médiévalité est telle dans lim

dans un décor de film telle dans l'imaginaire qu'on se projette dans un décor de film qui se joue au Moyen Âge. On se projette aussi dans les souvenirs d'enfance et les jeux de preux chevaliers : "On marche sur le rempart là c'est vraimx de preux chevajaime beaucoup vraiment je pense tout ce qui un endroit que un peu liés à l'enfance, des rêves de châteaux est les souvenirs cher sur le sommet du château je chateaux et tout ça de marson château. son château. " (Entretien itinérant $n^{\circ} 1$, couple.)

ÊTRE TÉMOIN DU “ ÇA A ÉTÉ " DE LA VIE DU UEU :

PARTAGER UNE HISTOIRE PERSONNELIE

La patrimonialité se construit aussi à partir de souvenirs d'enfance et celle-ci émerge, remonte lors de l'entretien. Il est alors important de souvenirs en refaisant avec les descendants ce que les ascendants ont fait avec le pratiquant ordinaire : "On m'y lle rocher des Doms a enec le à tous les âges de ma vie [ ] ' montait enfant au j'y ai fait j'y ai fait tous les jeux, j'y retrouvais toujours les mêmes petites amies // voilà c'était un peu notre jardin parce qu'on habitait place du Palais et on avait pas de jardin, peut-être que je me l'approprie plus que d'autres [...] le petit étang aux canards j'ai toujours été là à donner du pain aux canards on prenait du pain dur et on le donnait et je continue la tradition avec mes petitesfilles. "(Entretien $\mathrm{n}^{\circ} 14$, femme quatre-vingts ans.)

Les pratiquants ordinaires de la ville réactivent parfois une mémoire du lieu quand celui-ci a évolué et subi des transformations irréversibles : il se fait alors témoin d'un "ça a été ", acte photographique de l'objet patrimonial avant "l'accident ", c'està-dire le changement. On souhaite alors transmettre la connaissance du ça a été, réactiver la mémoire, l'état de quelque chose qui a existé, mais qui est inconsciemment oublié. Ce concept de mémoire réactivée par l'accident a été démontré par Sylvie Le Poulichet : " Après coup, une transmission a eu lieu lorsque dans la surprise s'est réalisé un acte de mémoire engageant au préalable les temps de l'oubli [...]. Mémoire et transmission s'effectuent simultanément à travers l'irruption de l'accident qui, en dernier ressort, s'identifie à la production d'un lieu de mémoire. " (Le Poulichet, 1990 : 170.) Les réactions des pratiquants face aux différents changer des actes de transmission dans la mesure où ils réactivent la mémoire du lieu et révèlent par là leur attachement à celui-ci. " Il y a un pin qui est mort, il a été touché par la foudre l'année dernière, il était maintenu par des étais et alors moi je voyais des pommes de pin qui étaient magnifiques tout en haut inaccessibles, et je pensais, j'avais entendu parler d'un chamane qui clisait de chercher à trouver l'inaccessible alors quand il est tombé j'ai ramassé des branches et des pignes et chaque année à Noël je sors ces branches et ces pommes de pin et je suis triste. "(Entretien $n^{\circ} 21$, femme, soixante-deux ans.)

Ils revendiquent même le droit de contester les changements d'un lieu qu'ils pratiquent au quotidien : "C'est la seule petite partie de remparts qu'on a le droit d'utiliser qui est ouverte au public c'est un peu dommage que depuis deux ans ils aient fermé la partie du bas qui rejoignait le pont, on a privé un peu le public de cette partie et ça je trouve que c'est dommage on a privatisé cette partie du rempart et du pont, ça faisait une liaison entre le pont et le jardin ce qui n'est plus, ça c'est pour les réclamations ! " s'indigner : l'entretien est l'occasion de faire passer un certain s'indigner : l'entretien est l'occasion de faire passer un certain
nombre d'idées, notamment critiquer la politique culturelle de la nombre d'idées, notamment critiquer la politique culturelle de la
ville, ou plutôt la non-politique de mise en valeur de centre historique : la ville abandonnerait son patrimoine. 


\section{$\mathrm{CONCL}$ O S I O N}

C

stefforcé de montrer l'intérêt de patrimonialité. Sa complexité, son côté sensible et impalpable, ténu et impensé, rend difficile la mise en place d'une méthode. Le défi est alors de trouver les outils qui permettent de repérer cette patrimonialité et de la rendre visible, lisible, donc verbalisable. Les allers-retours entre la constitution des outils et les premiers résultats ont permis de les réajuster en des éprouver en permanence sur le terrain. C'est ainsi que le dispositif de l'entretien itinérant, en donnant la parole aux pratiquants ordinaires, semble le plus heuristique en ce sens qu'il est une mise en discours de la ville par les habitants qui, pendant l'itinéance, prennent conscience de leurs pratiques et de leurs représentations vis-à-vis de la ville patrimoniale. Au fil des rencontes, nous avons pu repérer quelques indicateurs qui permettent construire le concept de patrimonial es pratiquants ordinaires construisent leur relation avec la ville patrimoniale, relation basée sur des pratiques, des représentations, des mémoires. Un des résultats de la recherche (toujours cours) est cet entremêlement de plusieurs valeurs, révélateur de l'ambiguiité de la notion de patrimoine, oscillant entre patrimoine individuel, issu de la famille et faisant app venirs et le patrimoin, in sou-

Mais peut-on réellement saisir toute la dimension de la patrimonialité et comprendre comment celle-ci émerge ? Nous restons persuadée que c'est dans l'articulation entre le verbatim le parcours in situ et l'étude des comportements que se joue la parpréhension de cette logique discursive. On voit se profiler une certaine organisation des pratiques et des perceptions.

A. W.

Laboratoire Culture \& Communication Université d'Avignon et des Pays de Vaucluse
6. L'expérience issue de la nouvelle économie, liée à l'économie de seréconomie, liée à l'économie de services et l'entertainment economy ou economie culturelle de divertissemen. Les pragnatistes anéricains comme John Dewey ont place lexpérience au centre de leurs theories Ce programme sur les émotion patrinoniales realise par le LAHIC va T Tor a 2006). \& Tornatore (dir.), 2006)

8. Emmanuel Ethis parle de - poïtique pour expliquer ce terme de fabrication (Ethis, 2004)

Nous désignons comme amateur de la ville un sujet n'habitant et ne travaillant pas à Avignon mais ayant ou ayant eu une pratique dAvignon. I a eu, par exemple, une pratique famille de lespace unbain relative au temps de lenfance.

10. Mathis Stock est géographe et a développé le concept de l'habiter dans sa thèse de doctorat (Stock, 2001). 11. Dans Les Cadres sociaux de la mé moire (1925), Maurice Halbwachs montre que le rappel du souvenir dépend du souvenir de Phomme avec l'es pace. Il met au jour - les relation entre l'existence collective, l'évocation individuelle de souvenirs et les rapports vécus avec l'espace * (Duvignaud, 1995).

12. L'avènement de la micro-histoire s'es fait dans les années 1970 en Italie, à l'initiative de Carlo Ginzburg et Giovanni Levi.

13. Jean-Jacques Boutaud et Pascal Lardellier prônent une sémio-anthropologie du sensible, c'est-à-dire la contribution de la sémiotique pou analyser le monde sensible (Boutaud \& Lardellier, 1973)

14. Les fameux parcours, type - fourmi - sauterelle, , - papillon,$\cdot$, poisson. (Véron \& Levasseur, 1983).

15. Certeau a repris à son compte les figures de cette rhétorique, qui son une analogie entre l'acte de marcher et l'acte de parler. Les deux figures phares construites sont celles de la synecdoque - procès par lequel, entre plusieurs éléments confrontés, une sélection s'effectue par laquelle on prend le tout pour la partie et la d'ancienneté, d'effets subjectifs et affectifs du monument. 
partie pour le tout - et l'asyndète
- liens par lesquels tout élément

d'expression en suit un autre dans la constitution de l'ensemble expressif (Augoyard, 1979).

16. Ces deux terminologies se retrouvent tant en muséologie qu'en sociologie urbaine.

17. Voir notamment la théorie du réci de Walter R. Fischer (1987).

18. Souligné par l'auteur dans le text original.
RÉFÉRENCES BIBLIOGRAPHIQUES

Amougou (Emmanuel) (dir.). 2004. La Question patrimoniale : De la * patrmonialisation *à l'examen des $s i$ tuations concrètes. Paris : Éd. de l'Harmattan.

Augoyard (Jean-François), 1979. Pas à pas : Essai de cheminement quotidien en milieu urbain. Paris : Ed. Seuil.

Augoyard (Jean-François). 2001. - La conduite de récit x p. 173-196 in L'Espace urbain en melbodes/ sous la direction de Michele Grosjean et Jean-Paul Thibaud. Marseille : Éd. Parenthèses (Eupalinos).

Barbe (Noel) \& Tornatore (Jean-Louis) (dir). 2006. Les Formats d'une cause patrimoniale : Émotions et action. autour du château de Lunéville rapport de la mission à l'ethnologie. Paris : ministère de la Culture et de la Communication

Barthes (Roland). 1993 [1964]. • La tou Eiffel , p. 1381-1400 in Essais critiques. Paris : Éd. du Seuil.

Boutaud (Jean Jacques) \& Lardellie (Pascal). 1973. • Sémio-anthropologie du sensible - Degrés, 113 , p. $1-4$ Certeau (Michel de), 1990. L'Invention du quotidien. t. I * Arts de faire * Paris : Gallimard

Da-Lage (Émilie) \& Gellereau, (Michèle). (À paraître) \& L'expert et l'amateur: valoriser l'interprétation du patrieur urbain par les habitants , contrimoine cation au colloque in communiLes Arts de la Ville dans la prospec tive urbaine, univins la prospec9-10 mars 2006

Davallon (Jean). 2006. Le Don du Patrimoine Pourune Le Don au Patrication Pour une approche communicalion Puris / Londres : Ed. Hermès Science Publications / Lavoisier. (Communications, médiations et construits

Davallon (Jean) (dir.). 2003. * Nouveaux regards sur le patrimoine Culture \& Musées,

Dondero (Maria Giulia). 2007. * Les pratiques photographiques du touriste entre construction d'identités et documentation ". Communication et Langages, 151, p. 21-38.

Duvignaud (Jean), 1995. Relecture d'Halbwachs : représentations collectives et valeurs de l'espace , in Ville, Fspace et Valeurs / sous la direction de Jean-Loup Gourdon, Evelyne Perrin \& Alain Tarrius. Paris : Éd, de l'Harmattan.

Ethis (Emmanuel). 2004 Le Spectateur imagé : Pour une poïétique de questionnaire. Paris : Éd de l'Harmatan. (Logiques sociales.)

Fischer (Walter R.). 1987. Human Communication as Narration : Toward a Pbilosophy of Reason, Value and Action Colombia : University of Action. Colombia : Carolina Press.

Guérin (Anne-Marie) 2004. Action publique locule et patrimoine culturel: Production et lesitimation des territoires politiques. Th sciences politipues: IIP de Grenoble. sciences poltiquis : 1.2. de Grenoble. sociaur de la ménoire Paris : Presses sociaux de la memoire. Pa

Jannerz (UIf) 1983. Explorer la ville:

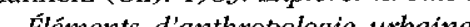
Paris : Éd. de Minuit.

Panis : Ed de Mrept

Hennion (Antoine). 2000. Figures de lamar delai. Paris: thal. Pans : Ed. de la Documentation fancise. (Question de culture.)

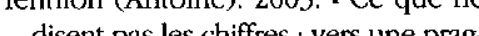
disent pas les chiffres : vers une pragmatique du gon -, in Regards crobes sur les pruncus culnur la Documentation française.

Jacquinot-Delaunay (Geneviève) \& MonJacquinot-Delaunay (Geneviève) \& Monnoyer (Laurence). 2000. * Introduction - Hermes, * Le dsposif : entre usage et concept $x, 25$

Jarrigeon (Anne). 2005. - Vers une poétique de lanonymat urbain $\cdot$. MEI, 21, te Poulichet (Martine). 1950. • Boufee de mémoire, p. 167-173 in Patrimoines en folles / sous la direction d'Henri-Pierre Jeudy. Paris : Ed. de la Maison des sciences de l'homme. (Ethnologie de la France)

Lynch (Kevin). 1971. L'Image de la cité tradult de lamericain par M.-F. e J.-L. Vénard. Paris : Dunod. (Aspects de l'urbanisme.) [Publication originale en 1960 .

riani-Rousset (Sophie), 1992. Les Parcours dexposition: Une situation de communication. Du compontement a la construction de sens. Th. doct. psychologie : université Lyon-2. Mariani-Rousset (Sophie). 2001. . I méthode des parcours dans les lieux d'exposition *, p. 29-44 in $L^{\prime} E s p a c e$ urbain en méthodes / sous la direction de Michèle Grosjean et Jean-Paul Thibaud. Marseille : Éd. Parenthèses (Eupalinos).

Mondada (Lorenza). 2000. Décrire la ville : La construction des savoirs urbains dans l'interaction et dans le texte. Paris : Éd. Anthropos.

Mondada (Lorenza). 2003. * Comment enquêter en ville et sur la ville? Urbanisme, - Imaginer, dire et faire la ville *, HS, 19, juil. 2003.

Montpetit (Raymond). 2005. . Expositions, parcs, sites : des lieux Expopériences patrimonias lieux dexÉ Musées, 5, p. 111-133.

Poggi (Marie-Hélène). 1999 - Le quartier du Marais : le mélange et le feuilleté in Pour une sociologie de la forme/ sous la direction de Nadir Marouf. Paris : É direction de Nadir Maten (Michel). 1998. L'émergence paich gence patrimoniale de l'ethnologie : bliques, p. 279-289 in Patrimoine et Modernité / sous la direction de Dom Doming (Chol. Pans : Ed. de PHarDon. des moneris : Son essence des monumb : Son assence el sa D. Wieczorek. Paris : Éd du Seuil D. We (Es 1903) T.) Tublica

ck (Mathis). 2001. Mobilités géographtques et pratiques des lieux: Etude thérico-empirique à travers deux

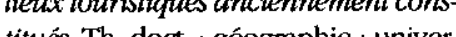
inues. Th doct. : gegraphie : vniversité Paris-7.

(Mathis). 2004. * L'habiter comme pratique des lieux géographiques :, <wWw. espacestemps.net/docu2007). 2007). 

(Eupalinos).

Vareille (Emmanuelle). 2001. L'Entre tien comme méthode et situation d'enquête: Le cas de l'évaluation muséale. Th doct. sciences de l'information et de la communication université d'Avignon et des Pays de Vhuchse. Vaucluse.

1983. Le) \& Levasseur (Martine) 1983. L'Espace, le corps, le sens: Ethnograpbie d une exposilion. Paris che des Eudes et de la Recherche de la Bl/ Cente Geor-

Veyne (Paul). 1988. * Conduites sans croyance et œuvres dart sans spectateurs x. Diogène, 143, p. 3-22. de l'opérationnalisation d'un concept, celui de patrimonialité, par la mise en place d'une méthodologie qualitative. Nous définissons la patrimonialité comme le rapport d'attachement des pratiquants ordinaires d'une ville avec ce qu'ils considèrent comme leur patrimoine. Au cour de la recherche il y a un quescomme leur patrimoine. Au cœur de la recherche il y a un questionnement méthodologique. Elle se veut comme une construc-
tion théorique pour comprendre la formation ordinaire du regard patrimonial dans un espace urbain particulier, le centre historique de la ville d'Avignon. Nous avons ainsi élaboré une méthodologie et exploré un certain nombre d'outils permettant de repérer et d'étudier la patrimonialité et ses expressions, ses modalités d'existence dans les discours et les pratiques ordinaires de l'espace urbain. Le dispositif de l'entretien itinérant réalisé dans le centre historique d'Avignon a plusieurs atouts : il fait force de preuve par la mise en présence de l'interviewé dans les lieux, il permet de construire un récit tout en parcourant la ville, il permet d'avoir accès aux pratiques quotidienmes, ordinaires de la ville. Grâce a lui, on peut comprendre comment le pratiquant ordinaire construit son attachement au patrimoine urbain à travers les pratiques ordinaires. L'analyse de certains entretiens réalisés lors de l'itinérance a dégagé quelques indicateurs de patrimonialité qui permettent de comprendre les représentations patrimoniales à partir du sujet. De la parole des individus, on peut comprendre que la patrimonialité est une certaine manière de nouer une relation, une expérience, un attachement avec un objet patrimonial reconnu ou ordinaire.

\section{$T_{b}$}

operationnalisation of a concept that of patrimonity of the patrimoniality, by setting up a qualitative methodology. We define patrimoniality as the act of attachment of ordinary users in a town with what they regard as their beritage. At the center of this research there is a methodological reflexion. It wants to be a theoretical construction in order to understand the ordinary creation of how one regards one's beritage in a particular urban space, in this case the bistorical center of the town of Avignon. We this elaborated a metbodology and explored a certain number of tools allowing us to locate and study the patrimoniality and its representations, its methods of existence in the discourse and the ordinary practices of urban space. The device of the itinerant interview carried out in the bistorical centre of Avignon has several assets : it makes force of proof by the setting in the presence of the person interviewed in sing the city, it makes it possible to have access to the daily, ordinary 